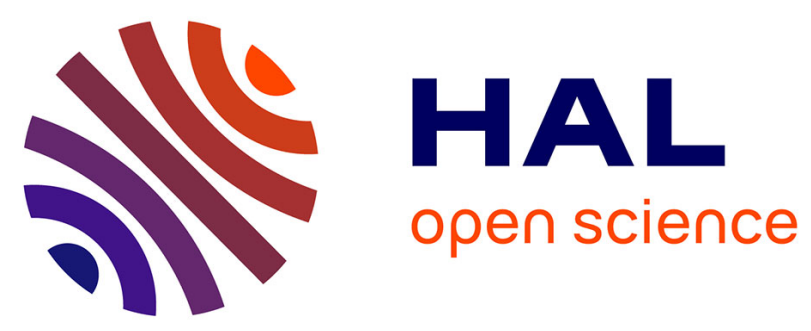

\title{
Etude micromécanique de la disparition du seuil de fissuration par fatigue dans un alliage de titane biphasé à fort Kmax
}

\author{
F. Mignot, V. Doquet, C. Sarrazin-Baudoux, J. Petit
}

\section{- To cite this version:}

F. Mignot, V. Doquet, C. Sarrazin-Baudoux, J. Petit. Etude micromécanique de la disparition du seuil de fissuration par fatigue dans un alliage de titane biphasé à fort Kmax. Journal de Physique IV Proceedings, 2003, 106, pp.33-42. 10.1051/jp4:20030213 . hal-00111377

\author{
HAL Id: hal-00111377 \\ https://hal.science/hal-00111377
}

Submitted on 23 Mar 2021

HAL is a multi-disciplinary open access archive for the deposit and dissemination of scientific research documents, whether they are published or not. The documents may come from teaching and research institutions in France or abroad, or from public or private research centers.
L'archive ouverte pluridisciplinaire HAL, est destinée au dépôt et à la diffusion de documents scientifiques de niveau recherche, publiés ou non, émanant des établissements d'enseignement et de recherche français ou étrangers, des laboratoires publics ou privés.

\section{(c)(1)}

Distributed under a Creative Commons Attribution| 4.0 International License 


\title{
Étude micromécanique de la disparition du seuil de fissuration par fatigue d'un alliage de titane biphasé à fort $K_{\max }$
}

\author{
F. Mignot, V. Doquet, C. Sarrazin-Baudoux ${ }^{1}$ et J. Petit ${ }^{1}$ \\ LMS, École Polytechnique, 91128 Palaiseau cedex, France \\ ${ }^{1}$ LMPM, ENSMA, 86961 Futuroscope cedex, France
}

\begin{abstract}
Résumé : L'alliage Ti6246 présente, lors d'essais de fissuration par fatigue à $\mathrm{K}_{\max }$ constant et $\Delta \mathrm{K}$ décroissant un comportement atypique : lorsque $\mathrm{K}_{\max } \geq 0.7 \mathrm{~K}_{\mathrm{IC}}$, on observe, sous air comme sous vide, au lieu du seuil attendu, un régime de propagation à vitesse constante, dix fois plus élevée à l'ambiante qu'à $500^{\circ} \mathrm{C}$. Le fluage, qui se manifeste dès l'ambiante dans cet alliage semble jouer un rôle dans ce phénomène. Toutefois, des analyses par spectrométrie de masse d'ions secondaires font apparaître une sur-concentration en hydrogène au voisinage d'une fissure développée dans le régime anormal. Des essais de fissuration, traction et fluage sont donc entrepris sur le matériau brut, appauvri ou enrichi en hydrogène, pour partie dans la chambre d'un microscope électronique à balayage, dans le but d'explorer ce phénomène. On constate qu'un enrichissement en hydrogène augmente la ductilité du matériau et sa tendance au fluage à l'ambiante et un enrichissement plus prononcé la diminue.
\end{abstract}

\section{INTRODUCTION}

La connaissance du seuil de fissuration par fatigue des matériaux de structure est essentielle pour la maintenance de pièces «tolérantes au dommage ». La détermination de ce seuil nécessite des essais de fissuration sous une amplitude $\Delta \mathrm{K}$ décroissante, jusqu'à ce que la vitesse de propagation de la fissure devienne inférieure à $10^{-10} \mathrm{~m} /$ cycle. Les essais à $\mathrm{K}_{\max }$ constant et $\mathrm{K}_{\min }$ croissant sont de plus en plus souvent préférés aux essais à rapport $\mathrm{R}=\mathrm{K}_{\min } / \mathrm{K}_{\max }$ constant, puisqu'ils permettent, si $\mathrm{K}_{\max }$ est suffisamment grand, de s'affranchir des effets de fermeture (si $\mathrm{K}_{\max } \geq \mathrm{K}_{\text {ouverture, }}$ le seuil déterminé est bien intrinsèque). En outre, pour les alliages constituants des pièces de moteurs aéronautiques, de tels essais se rapprochent des chargements en service, caractérisés par des fluctuations de faible amplitude autour d'une contrainte moyenne élevée.

Pour la plupart des matériaux, les seuils de fissuration et les lois de Paris déterminés avec la méthode conventionnelle ( $\mathrm{R}$ constant) ou la méthode à $\mathrm{K}_{\max }$ constant sont identiques. Toutefois, dans certains alliages de titane, si $\mathrm{K}_{\max }$ excède 60 à $70 \%$ de la ténacité, on observe, lorsque $\Delta \mathrm{K}$ décroît, non pas une décroissance de la vitesse de fissuration mais une propagation à vitesse sensiblement constante, qui ne dépend que de $\mathrm{K}_{\max }$ (Figure 1a) [1-4]. Dans l'alliage Ti6246, l'anomalie a été observée sous air comme sous vide secondaire (Figure lb) et ne résulte donc pas d'une interaction avec l'environnement externe. Le fluage, présent dès la température ambiante dans cet alliage, pourrait en partie expliquer le phénomène. C'est ce que suggère la figure $2 \mathrm{a}$ qui compare les cinétiques obtenues à $20^{\circ} \mathrm{C}$, lors d'essais menés à 3.5 et $35 \mathrm{~Hz}$. Pour $\Delta \mathrm{K} \geq 6 \mathrm{MPa} ل_{\mathrm{m}}$, les vitesses de propagation se corrèlent de façon unique à $\Delta \mathrm{K}$, mais dans le régime anormal, il exisìe un rapport 1 entre les vitesses asymptotiques, ce qui implique des vitesses identiques en termes de da/dt. Notons toutefois que la vitesse asymptotique de propagation dans le régime anomal est environ dix fois plus grande à la température ambiante qu'à $500^{\circ} \mathrm{C}$, température à laquelle l'anomalie n'apparât pas sous vide 
secondaire (Figure $2 \mathrm{~b}$ ). Notons enfin qu'à $150^{\circ} \mathrm{C}$, aucune anomalie n'a été décelée lors d'un essai à $\mathrm{K}_{\max }=57 \mathrm{MPa} \sqrt{m}$.
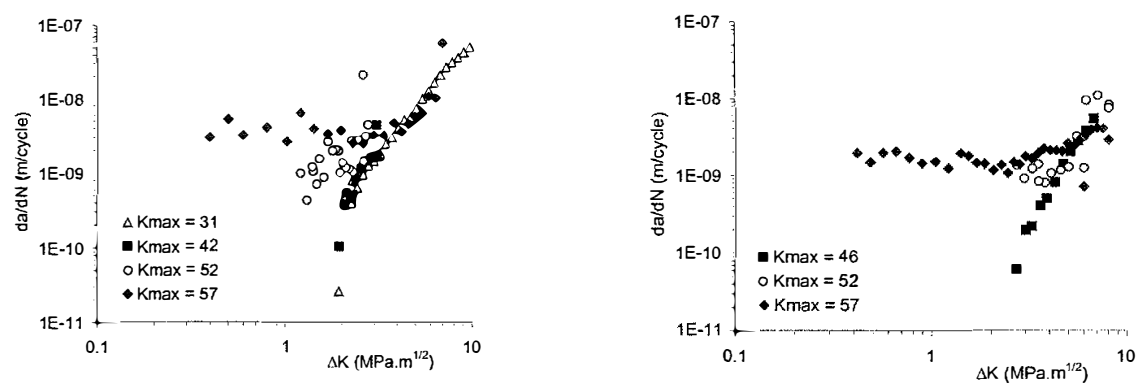

Figure 1 : Influence de $\mathrm{K}_{\max }$ sur le seuil de propagation en fatigue de l'alliage Ti6246 à $20^{\circ} \mathrm{C}$ (a) sous air (b) sous vide secondaire $[3,4]$.
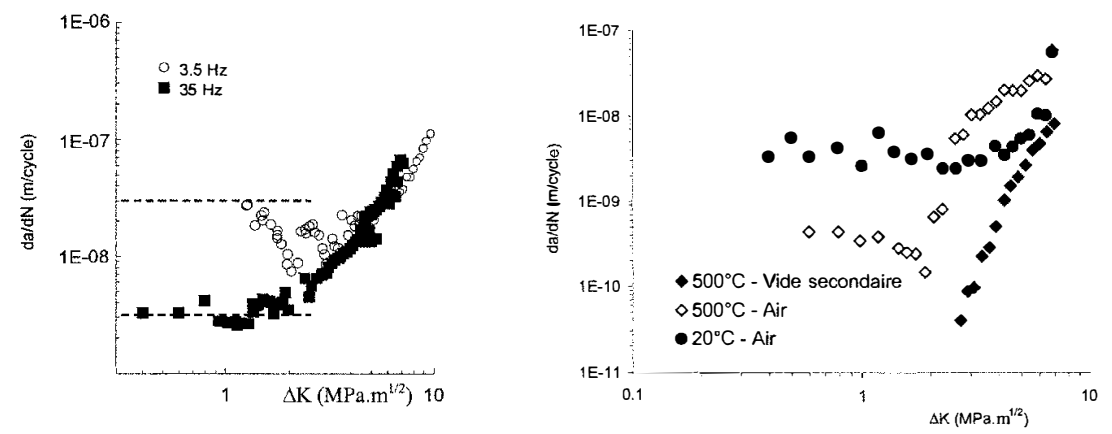

Figure 2 : (a) Influence de la fréquence de chargement à $20^{\circ} \mathrm{C}$ (b) Influence de la température sur la vitesse de fissuration par fatigue de l'alliage Ti6246 $\left(\mathrm{K}_{\max }=57 \mathrm{MPa} \sqrt{\mathrm{m}}\right.$, air $)$.

Ces éléments, associés à des surfaces de rupture particulières, comportant des facettes cristallographiques dans la phase $\alpha$ (Figure 3, quasi-clivage ?) et des ruptures le long des anciens joints de grains $\beta$, pour les essais menés à $20^{\circ} \mathrm{C}$, conduisent à soupçonner un effet de l'hydrogène interne qui se concentrerait autour de la pointe de la fissure, zone à fort taux de triaxialité des contraintes.

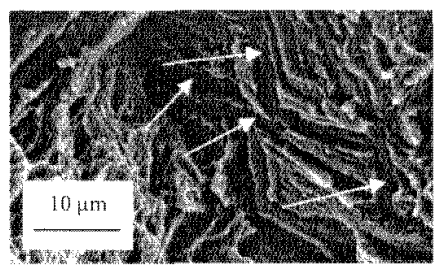

Figure 3 : Surface de rupture $\left(K_{\max }=57 \mathrm{MPa} \sqrt{\mathrm{fn}}, \Delta \mathrm{K}=2,20^{\circ} \mathrm{C}\right.$, sous air $)[3,4]$.

Les travaux de Shih et al [5] ont en effet montré que la fissuration du titane $\alpha$ est susceptible d'être accélérée par l'hydrogène selon deux mécanismes potentiels: précipitation et rupture d'hydrures de titane ou simple accroissement de la mobilité des dislocations favorisant le glissement localisé et la rupture selon des plans de glissement. Notons qu'a $500^{\circ} \mathrm{C}$, les 
surfaces de rupture correspondant au régime anormal de fissuration sont notablement différentes de celles observées à $20^{\circ} \mathrm{C}$ et suggèrent des mécanismes différents (intervention du fluage "classique», assisté par l'environnement, selon Sarrazin-Baudoux et col. $[3,4]$ ). L'analyse du phénomène à la température ambiante est plus problématique et constitue l'objectif de cette étude qui cherche en particulier à cemer les rôles respectifs du fluage à froid et de l'hydrogène interne.

\section{2. ÉTUDE EXPERIMENTALE}

\subsection{Matériau et procédures expérimentales}

\subsubsection{Le matériau et les traitements thermiques}

Le matériau étudié ( $6 \% \mathrm{Al}, 2 \% \mathrm{Sn}, 4 \% \mathrm{Zr}, 6 \% \mathrm{Mo}, 1 \% \mathrm{O}, 40 \mathrm{ppm} \mathrm{N})$ est issu d'un rouet centrifuge d'hélicoptère, forgé dans le domaine $\beta$. Après mise en solution dans le domaine $\alpha$ $+\beta$, un revenu de vieillissement de 8 heures à $595^{\circ} \mathrm{C}$ est pratiqué. Il en résulte une microstructure de type Widmanstätten (figure 4) avec un enchevêtrement de lamelles $\alpha$ (de 10 à $30 \mu \mathrm{m}$ de long, 1 à $2 \mu \mathrm{m}$ d'épaisseur et de structure hexagonale compacte) dans une matrice $\beta$ (cubique centrée) contenant elle-même de fines lamelles $\alpha$ secondaires. La proportion totale de phase $\alpha$ est d'environ $75 \%$ et le diamètre des anciens grains $\beta$ est de l'ordre de $300 \mu \mathrm{m}$. La teneur globale en hydrogène varie de 30 à $80 \mathrm{ppm}$ en poids selon la zone de prélèvement dans le rouet.
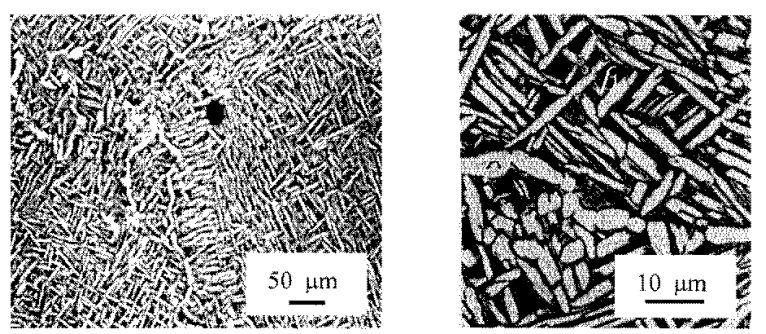

Figure 4 : Microstructure de type Widmanstätten de la nuance Ti6246.

Les principales caractéristiques mécaniques de l'alliage sont rassemblées dans le tableau 1.

Tableau 1 : Principales caractéristiques mécaniques à $25^{\circ} \mathrm{C}$ de l'alliage Ti6246

\begin{tabular}{|c|c|c|c|c|}
\hline$\sigma_{\mathrm{v}}(\mathrm{MPa})$ & $\sigma_{\mathrm{u}}(\mathrm{MPa})$ & $\mathrm{A}(\%)$ & $\mathrm{E}(\mathrm{GPa})$ & $\mathrm{K}_{\mathrm{lc}}(\mathrm{MPa} \backslash \mathrm{m})$ \\
\hline 860 & 1260 & 8 & 112 & 72 \\
\hline
\end{tabular}

Deux types d'éprouvettes ont été utilisés: des éprouvettes CTS de $3 * 23 * 37 \mathrm{~mm}$ pour les essais de fissuration et des éprouvettes plates de $4^{*} 1 \mathrm{~mm}$ de section et $30 \mathrm{~mm}$ de partie utile, pour le fluage ou la traction.

Une éprouvette CTS a été recuite sous vide secondaire pendant 500 heures, à $500^{\circ} \mathrm{C}$ (température ne semblant pas induire de modification microstructurale indésirable [6]). Sa teneur résiduelle en hydrogène, déterminée par des mesures de conductibilité thermique des gaz de fusion, est inférieure à $8 \pm 2 \mathrm{ppm}$ en poids. La teneur en oxygène et azote demeure inchangée. 
Deux protocoles destinés à charger le matériau en hydrogène ont été employés. Deux éprouvettes CTS ont subi un recuit d'une centaine d'heures à $500^{\circ} \mathrm{C}$ sous un flux d'argon $+10 \%$ d'hydrogène. Ce type de traitement s'est avéré efficace (teneurs finales d'environ $900 \pm 20 \mathrm{ppm}$ ) mais peu reproductible et peu approprié à l'introduction de faibles doses d'hydrogène. Des éprouvettes plates de traction et fluage ont subi un recuit d'une quarantaine $\mathrm{d}$ 'heures à $400^{\circ} \mathrm{C}$ dans une enceinte sous vide où a été introduite une dose finie d'hydrogène (pressions initiales de 10, 20 ou 40 mbar). Ces recuits comportent une phase d'absorption de l'hydrogène par l'échantillon (la pression d'hydrogène dans l'enceinte chute en 20heures environ) prolongée par une phase d'homogénéisation d'une vingtaine d'heures (au cours de laquelle on s'assure que la pression ne remonte pas). Les teneurs en hydrogène mesurées par analyse des gaz de fusion sont de $496 \pm 24,762 \pm 15$ et $1283 \pm 20$ ppm en poids, respectivement.

\subsubsection{Les essais mécaniques}

Les essais mécaniques sont menés en partie sur des machines conventionnelles et en partie sur des machines fonctionnant dans la chambre d'un microscope électronique à balayage.

Des microgrilles d'or de 2 à $4 \mu \mathrm{m}$ de pas sont déposées à la surface des éprouvettes par microlithographie électronique. Des techniques de corrélation d'images numériques de haute résolution acquises à différentes étapes des essais permettent d'accéder au champ local de déformation [7] lors des essais in situ.

Les essais de fissuration par fatigue sont menés à $15 \mathrm{~Hz}$. Après la préfissuration à rapport $\mathrm{R}$ faible, le cyclage est effectué à $K_{\max }$ constant et $\Delta \mathrm{K}$ décroissant par paliers de $8 \%$. Le suivi cinétique de la propagation est assuré, avec une résolution d'environ $10 \mu \mathrm{m}$, à l'aide d'un microscope optique.

\subsubsection{Analyses SIMS}

Contrairement aux techniques conventionnelles d'analyse, la spectrométrie de masse d'ions secondaires (SIMS) est appropriée au dosage d'éléments légers comme l'hydrogène, même à très faible teneur et avec une bonne résolution spatiale. Cette technique a donc été employée pour rechercher une éventuelle surconcentration en hydrogène au voisinage d'une fissure de fatigue, dans le matériau brut. Une coupe longitudinale à mi-épaisseur d'une éprouvette CT de $10 \mathrm{~mm}$ d'épaisseur préalablement fissurée par fatigue à fort $\mathrm{K}_{\max }$ jusqu'au régime anormal, a été polie de façon à pratiquer les analyses SIMS dans une zone ayant été soumise à un fort taux de triaxialité (zone de déformation plane). Quelques analyses pratiquées sur la surface externe de la même éprouvette ayant donné des résultats très voisins permettent de penser que la relaxation des contraintes induite par la découpe n'affecte pas trop la distribution de l'hydrogène. Un faisceau primaire d'ions $\mathrm{O}_{2}{ }^{+}$accéléré sous $20 \mathrm{kV}$, avec un diaphragme d'analyse de $8 \mu \mathrm{m}$ a été utilisé . Les mesures n'ont été prises en compte qu'après 3 minutes de bombardement ionique destiné à éliminer toute contamination superficielle (les cratères produits font quelques microns de profondeur). L'index de concentration en ions hydrogène, exprimé en "nombre de coups par secondes" est normalisé par l'intensité du faisceau primaire, dont on prend ainsi en compte les fluctuations dans le temps (entre 35 et $50 \mathrm{nA}$ pour les analyses présentées ci-dessous ).

\subsection{Résultats expérimentaux}

\subsubsection{Concentration en hydrogène au voisinage d'une fissure de fatigue, à fort $K_{\max }$}

L'évolution de la teneur en hydrogène mesurée par SIMS devant la pcinte d'une fissure développée dans le matériau le base, lors d'un essai sous air, à $K_{\max }=57 \mathrm{MPa} / \mathrm{m}$ ế $\Delta \mathrm{K}$ 
décroissant, interrompu dans le régime anormal, pour $\Delta \mathrm{K}=0.5 \mathrm{MPa} \sqrt{\mathrm{m}}$ est présentée sur la figure 5. Près de la pointe de la fissure, la teneur en hydrogène est deux à trois fois supérieure à la teneur moyenne de l'alliage. Elle retrouve le niveau moyen à une distance d'environ $1.7 \mathrm{~mm}$. Il est difficile de comparer cette distance à la taille de la zone plastique qui n'est pas connue avec précision (l'estimation par la formule d'Irwin $(1 / \alpha \pi)\left(\mathrm{K}_{\max } / \sigma_{\mathrm{y}}\right)^{2}$ : environ $0.47 \mathrm{~mm}$ en contraintes planes $(\alpha=1)$ et $1.4 \mathrm{~mm}$ en déformations planes $(\alpha=3)$ pour $\left.\mathrm{K}_{\max }=57 \mathrm{MPa} \sqrt{\mathrm{n}}\right)$. Cette estimation ne teint compte ni de l'adoucissement cyclique du matériau ni surtout du fluage en pointe de fissure qui la sous-estime certainement).

La figure 6 présente une cartographie de la teneur en hydrogène en arrière de la pointe, dans la zone correspondant à la transition du régime normal de fissuration par fatigue au régime anormal (soit $\Delta \mathrm{K}=3 \mathrm{MPa} \sqrt{ }$, voir figure 6). Les mesures sur la fissure elle-même, affectées par sa légère ouverture, ne doivent pas être prises en compte (de même que les mesures obtenues au bord de la zone balayée). Par contre, on constate un enrichissement en hydrogène dans une bande d'environ $50 \mu \mathrm{m}$ de large, de part et d'autre de la fissure, le long de la portion correspondant au régime anormal. On peut suggérer que l'absence apparente d'enrichissement dans la portion correspondant au régime normal de fissuration (c'est à dire à plus fort $\Delta K$ ) est due aux décharges et aux passages périodiques en compression qui rendent la pointe de fissure moins attractive pour l'hydrogène.

Il est en tout cas essentiel d'étudier comment une variation de la teneur en hydrogène de l'alliage affecte ses propriétés mécaniques.

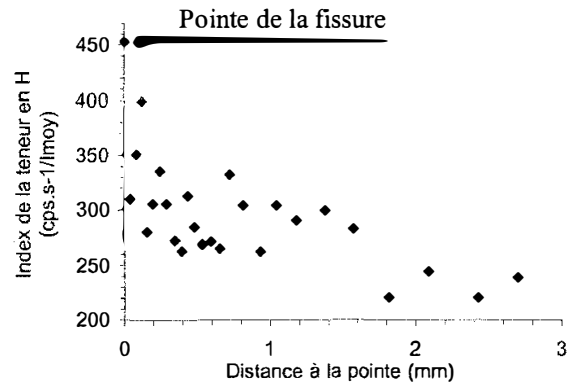

Figure 5 : Évolution de la teneur en hydrogène mesurée par SIMS en avant de la pointe d'une fissure de fatigue $\left(\mathrm{K}_{\max }=57 \mathrm{MPa} \sqrt{\mathrm{m}}, \mathrm{T}=20^{\circ} \mathrm{C}\right.$, sous air $)$

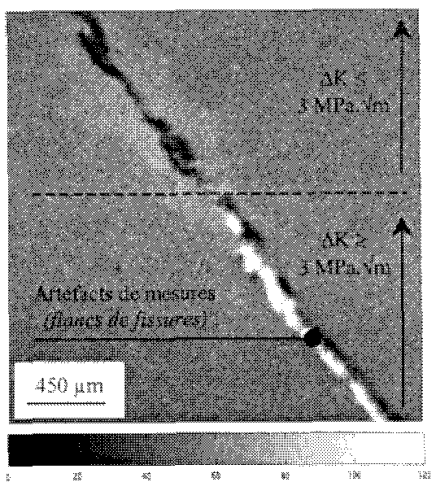

Figure 6 : Cartographie SIMS de la teneur en hydrogène dans la zone de ransition du régime de Paris au régime anormal $\left(\mathrm{K}_{\max }=57 \mathrm{MPa} \sqrt{\mathrm{m}}, \mathrm{T}=20^{\circ} \mathrm{C}\right.$, sous air $)$.

\subsubsection{Essais de traction in situ}

La figure 7 compare les courbes contrainte-déformation globale obtenues lors d'essais de traction sous MEB pour une vitesse de déformation de $6.10^{-5} \mathrm{~s}^{-1}$, sur le matériau brut ou chargé à $500 \mathrm{ppm}$ d'hydrogène (selon le deuxième procédé, à $400^{\circ} \mathrm{C}$ décrit au paragraphe 2.1). Ce chargement accroît de façon spectaculaire la ductilité de l'alliage.

A l'échelle locale, la déformation est très hétérogène, dans le matériau chargé comme dans le matériau brut (Figures $8 \mathrm{a}$ et $8 \mathrm{~b}$ ). Elle se localise dans des bandes à $45^{\circ}$ de l'axe de traction où la déformation équivalente de Von Mises peut atteindre 20\% (alors que la déformation axiale globale n'est que de 5.7\%). Certaines zones, au contraire, sont à peine déformées. Mais si 
cette déformation s'accompagne de l'apparition de lignes de glissement très marquées dans certaines lattes $\alpha$ du matériau de base, la déformation de la phase $\alpha$ semble plus homogène dans l'éprouvette chargée en hydrogène, où le glissement se localise plutôt aux interfaces $\alpha / \beta$ (Figure $9 \mathrm{a}$ et $9 \mathrm{~b}$ ). Des essais similaires sont en cours sur des éprouvettes plus fortement chargées en hydrogène.

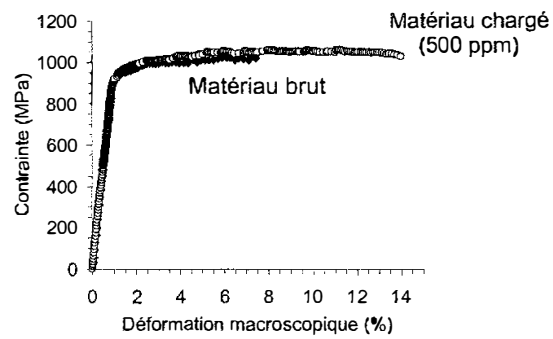

Figure 7 : Comportement en traction du matériau brut ou chargé en hydrogène (500 ppm).

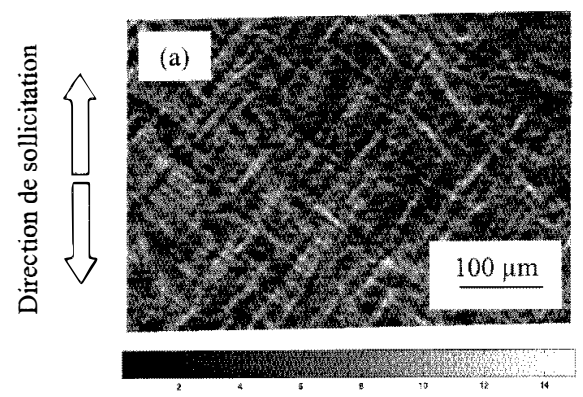

(b)

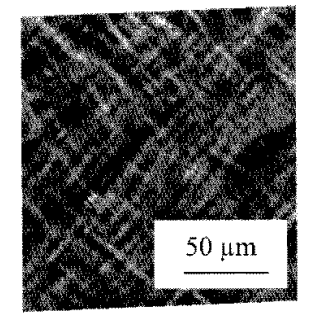

Figure 8 : Cartographies de la déformation équivalente de Von Mises. $\varepsilon_{\text {macro }}=5,7 \%$ (a) Matériau brut (b) Matériau chargé en hydrogène (500 ppm).
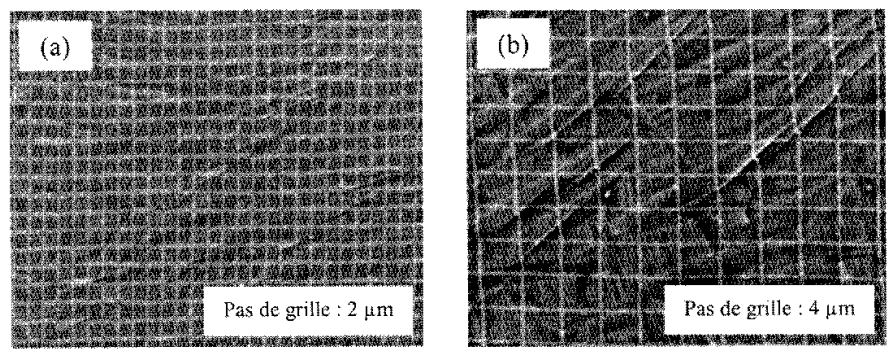

Figure 9 : Mécanismes de déformation associés (a) Matériau brut (b) Matériau chargé en hydrogène (500 ppm).

\subsubsection{Essais de fluage}

La figure 10 compare les courbes de fluage obtenues à $20^{\circ} \mathrm{C}$ sur des éprouvettes plates brute et chargées en hydrogène à 750 et $1280 \mathrm{ppm}$. La contrainte appliquée, $1000 \mathrm{MPa}$, est représentative du niveau de sollicitation, quelques dizaines de microns devant la pointe d'une fissure de fatigue, à fort $K_{\max }$, tel qu'il a pu être estimé par éléments finis. On constate qu'en quelques heures, la déformation atteint $2,5 \%$ dans le matériau brut. Le fluage est plus rapide 
dans l'échantillon chargé à 750 ppm et plus limité dans le matériau chargé à 1280 ppm. Des essais complémentaires sont en cours pour des teneurs intermédiaires afin de vérifier si la vitesse de fluage passe effectivement par un maximum lorsque la concentration en hydrogène augmente comme semblent le suggérer ces premiers résultats.
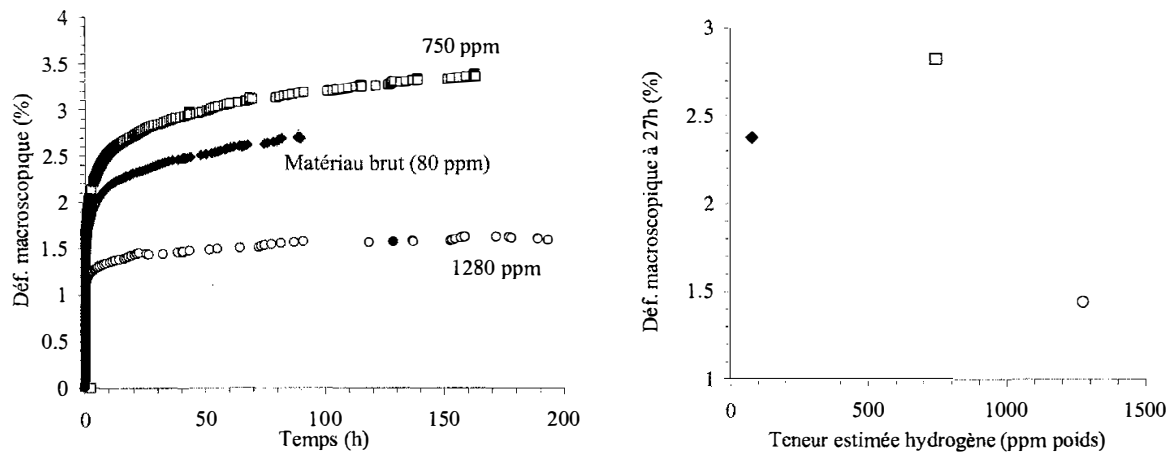

Figure 10 : Influence de la teneur en hydrogène sur le fluage à $20^{\circ} \mathrm{C}$ sous $1000 \mathrm{MPa}$

\subsubsection{Essais de fissuration}

La figure 11 rassemble les données cinétiques de fissuration par fatigue à $K_{\max }$ constant et $\Delta \mathrm{K}$ décroissant obtenues sur des éprouvettes CTS à diverses teneur d'hydrogène. Pour $K_{\max }=57$ $\mathrm{MPa} \sqrt{\mathrm{m}}$ dans le matériau de base, même s'il n'apparaît pas de véritable plateau sur la courbe cinétique tel qu'ont pu en observer Sarrazin et al.[3,4] lors d'essais sur des éprouvette CT de $10 \mathrm{~mm}$ d'épaisseur, dans lesquelles le taux de triaxialité des contraintes (favorable à la concentration d'hydrogène comme à l'endommagement par fluage) est plus élevé, on constate néanmoins que la fissuration se poursuit, pour des valeurs de $\Delta \mathrm{K}$ bien inférieures au seuil d'environ 3.5 $\mathrm{MPa} \sqrt{\mathrm{m}}$ mesuré lors d'essais à faible $\mathrm{K}_{\max }$, en régime «normal ».

Malgré une certaine dispersion des données cinétiques, on constate que dans l'échantillon appauvri en hydrogène, un seuil de non propagation semble être atteint pour $K_{\max }=62$ et même $67 \mathrm{MPa} \sqrt{\mathrm{m}}$. Au contraire, le régime anormal de fissuration est observé pour $\mathrm{K}_{\max }=20$ $\mathrm{MPa} \sqrt{\mathrm{m}}$ seulement, sur une éprouvette enrichie jusqu'à $900 \mathrm{ppm}$ en poids d'hydrogène (par recuit à $500^{\circ} \mathrm{C}$ sous $\mathrm{Ar} / \mathrm{H}$ ). En outre, la vitesse de propagation, quasi indépendante de $\Delta \mathrm{K}$, est dans ce cas près de 2 ordres de grandeur plus élevée que pour le matériau brut, à $K_{\max }=57$ $\mathrm{MPa} \sqrt{\mathrm{m}}$.

Un essai mené à l'air, à $\mathrm{K}_{\max }=57 \mathrm{MPa} \cdot \sqrt{\mathrm{m}}$ et $\Delta \mathrm{K}$ décroissant sur une éprouvette CTS brute a conduit à un arrêt de la propagation. L'éprouvette a alors été chargée (par recuit à $500^{\circ} \mathrm{C}$ sous flux d'argon /hydrogène) jusqu'à une teneur globale de l'ordre de $850 \mathrm{ppm}$ et l'essai a été repris dans les mêmes conditions, mais sous MEB. Les figures $12 \mathrm{a}, 12 \mathrm{~b}$ et $12 \mathrm{c}$, prises à $\mathrm{K}=$ $40 \mathrm{MPa} \sqrt{\mathrm{m}}$, montrent que l'émoussement de la pointe s'accroît non seulement lorsque la charge augmente, mais aussi pendant les phases de maintien et que la fissure tend à se développer à environ $60^{\circ}$ de l'axe de traction. Sous l'effet d'un chargement cyclique $\left(\mathrm{K}_{\max }=57 \mathrm{MPa} \sqrt{\mathrm{m}}, \Delta \mathrm{K}=2 \mathrm{MPa} \sqrt{\mathrm{m}}\right)$, la pointe se réaffute (figure $12 \mathrm{~d}$ ) et la fissure retrouve progressivement une orientation normale à l'axe de traction. Toutefois, dès que la charge est maintenue à sa valeur maximale, l'émoussement croît à nouveau (figure 12e). La figure $12 \mathrm{f}$, obtenue par corrélation des images $12 \mathrm{~d}$ et $12 \mathrm{e}$, montre qu'en deux minutes de maintien, la déformation équivalente de Mises s'est accrue de près de $20 \%$ dans certaines lattes $\alpha$. Le 
chargement en hydrogène semble donc favoriser la déformation visqueuse du matériau. L'endommagement apparaît en avant de la pointe, bien au delà de la zone plastique cyclique (dont la dimension n'excède pas quelques microns), dans certaines lattes et aux interfaces $\alpha / \beta$ (figure 13a). La propagation procède par coalescence répétée avec cet endommagement.

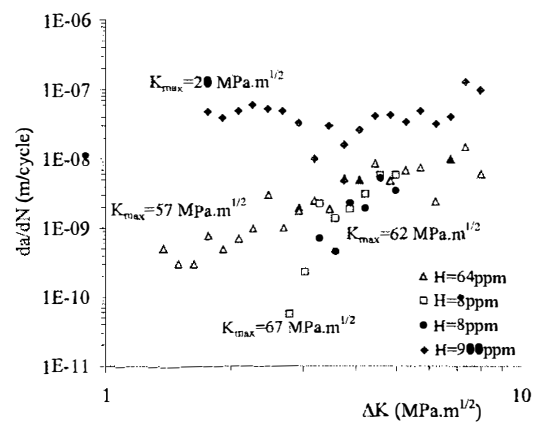

Figure 11 : Influence de la teneur en hydrogène sur la cinétique de fissuration par fatigue du Ti6246 (air, $20^{\circ} \mathrm{C}$ ).
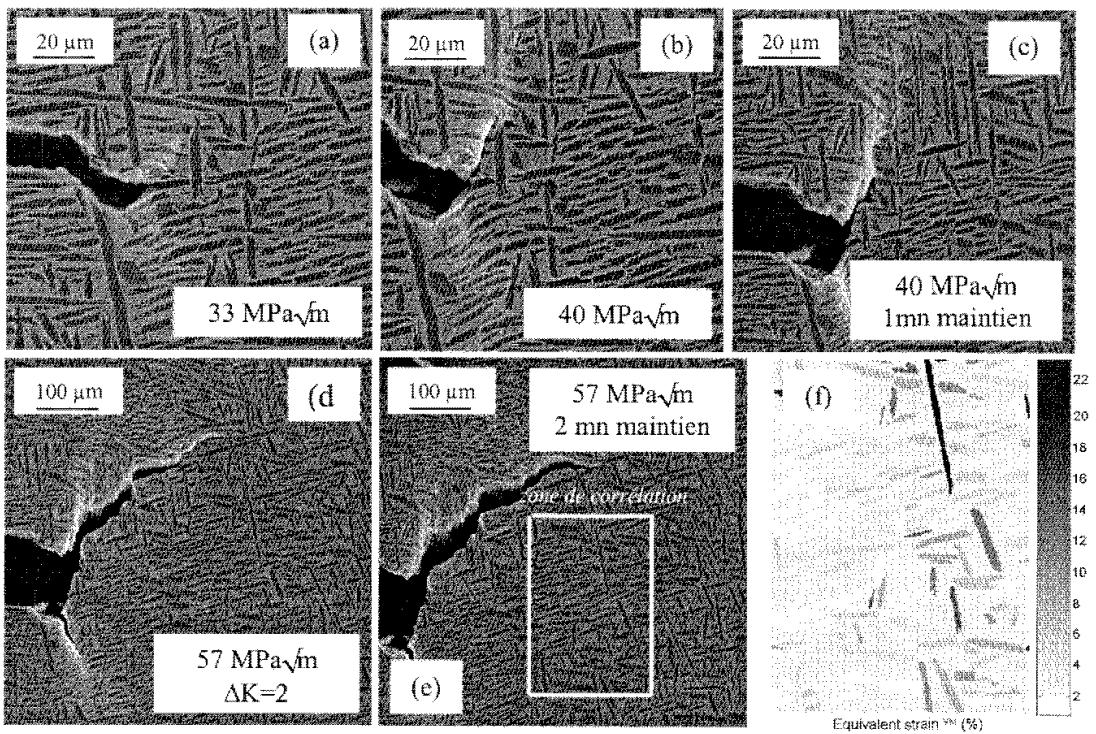

Figure 12 : Stades de la propagation d'une fissure dans une éprouvette CTS chargée en hydrogène.

La surface de rupture associée (Figure 13b) souligne la microstructure lamellaire. On y retrouve les facettes cristallographiques déjà observées sur le matériau brut dans le régime anormal (figure 3). Elle rappelle toutefois le « flutting » observé par Crépin et al. [8] dans un alliage de zirconium traité dans le domaine $\beta$. Ce type de faciès résulte alors de la croissance ductile de cavités prismatiques d'axe $<\mathrm{c}>$ et à section hexagonale, par activation des trois systèmes de glissement prismatique deux à deux. Des examens fractographiques plus poussés sont nécessaires pour préciser si les mécanismes de rupture dans le Ti6246 sont, ou non du même type. 


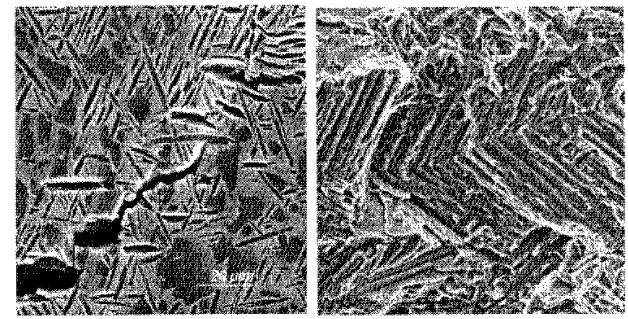

Figure 13 : (a) Endommagement des lattes $\alpha$ en avant de la pointe (b) Surface de rupture associée.

\section{DISCUSSION}

Les contributions respectives du fluage et de la «fragilisation » par l'hydrogène semblent difficilement séparables au vu des résultats expérimentaux, qui militent tantôt pour un mécanisme tantôt pour l'autre (sans exclure la possibilité d'un fluage assisté par l'hydrogène). Le fait que l'anomalie soit observée à $20^{\circ} \mathrm{C}$ et $500^{\circ} \mathrm{C}$ (quoique avec une vitesse asymptotique plus basse) mais pas à $150^{\circ} \mathrm{C}$ ne doit pas conduire à écarter l'hypothèse du fluage. Les travaux de Jousset et al [9] sur l'alliage Ti6242 ont en effet montré que, pour une même valeur de $\sigma_{\text {appliqué }} / \sigma_{y}(T)$, cet alliage ne présente qu'un fluage primaire à $150^{\circ} \mathrm{C}$, alors qu'à la température ambiante, le fluage ne se sature pas et conduit à la rupture. Les auteurs attribuent ce moindre fluage à des interactions entre dislocations et atomes interstitiels (oxygène ou azote). La sensibilité aux temps de maintien en fatigue («dwell effect») commune à de nombreux alliages de titane, s'atténue généralement aux alentours de $150^{\circ} \mathrm{C}$.

D'autre part, l'interprétation proposée par Lang [2] de la vitesse asymptotique plus faible à $500^{\circ} \mathrm{C}$ qu'à l'ambiante est basée sur une répartition différente de l'hydrogène au sein du matériau. A $20^{\circ} \mathrm{C}$, l'hydrogène serait piégé aux interfaces, favorisant la décohésion interfaciale. A $500^{\circ} \mathrm{C}$, sa diffusion dans les grains favoriserait la rupture ductile.

Rappelons en outre que la précipitation d'hydrures de titane, souvent invoquée pour expliquer la fissuration sous-critique sous charge constante (Sustained Load Cracking) n'est guère probable à $150^{\circ} \mathrm{C}$ et au delà. A $20^{\circ} \mathrm{C}$, compte tenu du degré d'enrichissement local en hydrogène mesuré en pointe de fissure par SIMS (un facteur 2 à 3), et de la très faible solubilité de l'hydrogène dans la phase $\alpha$, on ne peut l'exclure. L'observation au MET de lames minces prélevées en pointe de fissure permettra peut-être de mettre en évidence d'éventuels hydrures.

Par ailleurs, de nouvelles analyses par SIMS de la teneur en hydrogène seront pratiquées sur une éprouvette CT ayant été sollicitée sous vide secondaire, ce qui devrait permettre de préciser les contributions respectives du transport d'hydrogène interne vers la pointe de fissure et l'entrée d'hydrogène liée à l'environnement.

Sarrazin-Baudoux et al $[3,4]$ mettent en évidence un temps d'incubation précédent le démarrage de la fissuration sous charge constante lorsqu'après des sollicitations cycliques, $\mathrm{K}$ est brusquement figé à sa valeur maximale (figure 14). Le temps d'incubation, nul pour les très faibles valeurs de $\Delta K$, augmente ensuite avec $\Delta K$ et semble saturer. L'observation d'émoussement croissant de la fissure lors des phases de maintien et de ré-affutage lors des phases de chargements cycliques suggèrent que ce temps d'incubation pourrait correspondre au temps nécessaire pour atteindre un émoussement ou une déformation de fluage critiques devant la pointe. En effet, plus le $\Delta \mathrm{K}$ du chargement cyclique préalable est important, plus la plasticité en retour ou le simple effet de décharges substantielles à chaque cycle limite la déformation accumulée par fluage et retardent l'instant où la valeur critique sera atteinte. Des 
calculs par éléments finis en viscoplasticité sont en cours pour simuler la transition entre un chargement cyclique et un maintien. On ne peut toutefois exclure que le temps d'incubation corresponde au temps nécessaire pour atteindre une concentration critique en hydrogène sachant que cette demière est d'emblée assez élevée à faible $\Delta \mathrm{K}$, comme le montrent les mesures par SIMS.
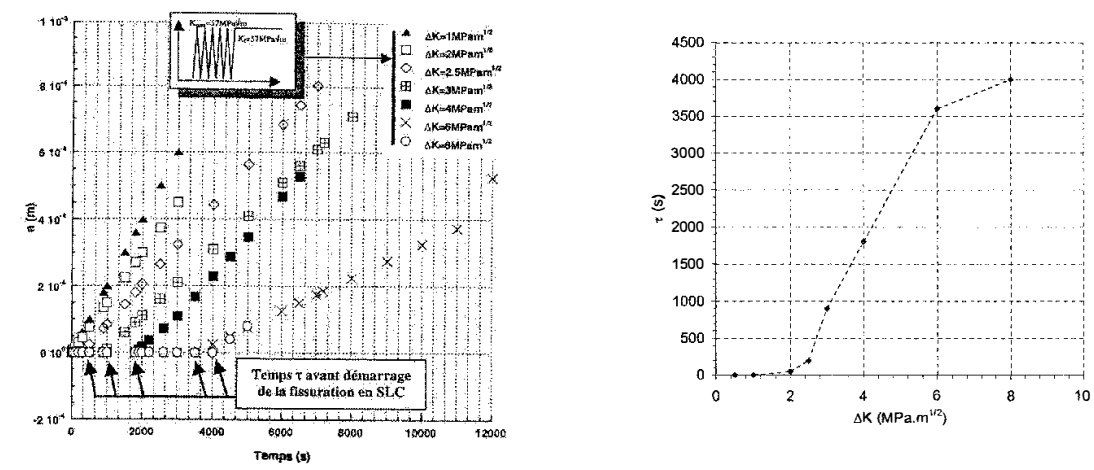

Figure 14 : Mise en évidence d'un temps d'incubation avant le démarrage de la fissuration sous charge constante après chargement cyclique $\left(\mathrm{K}_{\max }=57 \mathrm{MPa} \sqrt{\mathrm{n}}, \mathrm{T}=20^{\circ} \mathrm{C}\right.$, sous air). D'après Sarrazin-Baudoux et al $[3,4]$.

\section{Remerciements}

Les auteurs souhaitent remercier P. Doumalin et D. Caldemaison (LMS, école Polytechnique) pour leur aide sur les techniques de corrélation d'images numériques ainsi que A.-M. Brass (LPCES , Univ. Paris XI) pour les dosages d'hydrogène, L. Raimbault (LIG, ENSMP) pour les mesures SIMS et F. Garnier (LMSS-Mat, ECP) pour les observations au MEB-FEG.

\section{Références}

1. G. Marci, Fatigue 96, p. $493-498$ (1996).

2. M. Lang, Acta Mat. 47, 11, p. 3247-3261 (1999).

3. C. Sarrazin-Baudoux, Y. Chabanne, J. Petit, Scripta Mat., 40nº 4, p. 451-457 (1999).

4. C. Sarrazin-Baudoux, Y. Chabanne, J. Petit, ASTM STP 1372, p. 341-360, J.C. Newman Jr.,R.S. Piasick Eds., Philadelphia (2000).

5. D. Shih, I.M. Robertson, H.K. Bimbaum, Acta Met., 36, p. 111-124 (1988).

6. M. Bourgeois, X. Feaugas, M. Clavel, Scripta Met., 34, 9, p. 1483-1490 (1996).

7. P. Doumalin, M. Bornert in Interferometry in speckle light, theory and applications, edited by P.Jacquot and J.M.Fournier, Springer, p. 67-74 (2000).

8. J. Crépin, T. Bretheau, D. Caldemaison, Cavity growth and rupture of $\beta$-treated zirconium : a crystallographic model. Acta Mater., 44, 12, p. 4927-4935, 1996.

9. H. Jousset, L. Remy, J.L. Strudel, ICM8, Victoria, 2, p. 580-585, edited by F. Ellyin and J.M. Provan, 1999. 